\title{
Strates
}

STRATES Matériaux pour la recherche en sciences sociales

9 | 1997

Crises et mutations des territoires

\section{Sentiers de trekking et activités de portage au Népal}

Isabelle Sacareau

\section{(2) OpenEdition}

1 Journals

Édition électronique

URL : http://journals.openedition.org/strates/622

DOI : $10.4000 /$ strates. 622

ISSN : $1777-5442$

Éditeur

Laboratoire Ladyss

Édition imprimée

Date de publication : 30 septembre 1997

ISSN : 0768-8067

Référence électronique

Isabelle Sacareau, «Sentiers de trekking et activités de portage au Népal », Strates [En ligne], 9 | 1997, mis en ligne le 19 octobre 2005, consulté le 07 septembre 2020. URL : http://journals.openedition.org/ strates/622 ; DOI : https://doi.org/10.4000/strates.622

Ce document a été généré automatiquement le 7 septembre 2020

Tous droits réservés 


\title{
Sentiers de trekking et activités de portage au Népal
}

\author{
Isabelle Sacareau
}

1 Le sentier est sans doute la trace linéaire la plus ancienne, mais aussi la plus fine et la plus discrète que les hommes aient imprimée sur leur espace. A l'ère des autoroutes et des trains à grande vitesse, ils n'apparaissent plus comme des éléments capables de structurer l'espace ou de conserver une fonction économique, même si le tourisme «vert » en redécouvre les vertus, à travers le nouvel engouement pour la randonnée pédestre. Pourtant, dans certains pays montagneux et enclavés du Tiers-monde qui ne disposent pas de moyens de transport modernes développés, les sentiers continuent de jouer un rôle économique et social fondamental. Malgré la faiblesse de leur empreinte spatiale et des flux qu'ils engendrent, ils sont un instrument essentiel des relations entre les hommes en zone rurale. Depuis une trentaine d'années, leur usage se transforme dans un certain nombre de pays avec l'apparition du tourisme de trekking.

Le Népal, petit royaume adossé à la chaîne himalayenne, présente un cas exemplaire de l'importance qu'une infrastructure de transport, aussi modeste soit-elle, peut tenir dans l'économie et la structuration de l'espace d'un pays pauvre. Malgré son altitude et les difficultés que son relief gigantesque oppose à la circulation, l'Himalaya n'a jamais été une barrière : points de contact entre les civilisations hindouistes et bouddhistes, les sentiers et les cols d'altitude du Népal ont été sillonnés de tout temps par les envahisseurs, les marchands ou les pèlerins. Le bassin de Kathmandou situé à $1300 \mathrm{~m}$ d'altitude a servi de point de rencontre des grandes routes caravanières entre l'Inde et le Tibet, animées par un flux incessant de porteurs et de convois de mules ou de yacks. Un réseau dense de sentiers couvre tout le pays où se pratique toujours le portage à dos d'homme. Aujourd'hui, avec le tourisme de trekking, ce sont des dizaines de milliers de touristes venus du monde entier qui empruntent ces chemins construits et entretenus depuis des siècles par les paysanneries himalayennes. S'appuyant sur ces infrastructures villageoises et sur la tradition de portage commercial des montagnards du Népal, le trekking a surimposé à ce réseau de sentiers ses propres itinéraires : il 
entraîne alors une nouvelle structuration de l'espace, en intégrant progressivement à l'économie moderne des massifs montagneux autrefois isolés.

Une civilisation de la marche

3 Au Népal, les déplacements se heurtent à de nombreux handicaps physiques : les deux tiers du pays sont constitués de chaînes parallèles de taille croissante culminant entre 2000 et plus de $8000 \mathrm{~m}$ d'altitude. La disposition latitudinale d'un relief accidenté, à peine aéré par quelques vallées transversales au profil irrégulier, des altitudes moyennes élevées et un climat marqué l'été par les violentes pluies de mousson, y rendent les communications particulièrement difficiles.

4 Pourtant, la montagne himalayenne est un milieu profondément humanisé. Les moyennes montagnes du pays concentrent près de la moitié de la population, le reste se localisant essentiellement sur le piémont himalayen dans la plaine du Teraï. Le peuplement est particulièrement dense dans les bassins du Moyen-Pays où se localise la capitale Kathmandou et la deuxième ville du pays, Pokhara. Il s'étage ensuite sur de longs versants aux puissants dénivelées entre 1500 et $2500 \mathrm{~m}$ d'altitude. Au-dessus, l'habitat permanent se niche en petits îlots discontinus dans les hautes vallées glaciaires du Grand Himalaya jusque vers $4500 \mathrm{~m}$.

5 La pauvreté structurelle de ce pays à $90 \%$ rural n'a pas permis d'y mettre en place des infrastructures routières suffisantes, capables de desservir et de mettre en valeur les rares ressources naturelles du pays, ni même de structurer un véritable réseau urbain. Celui-ci se réduit à Kathmandou la capitale du pays, à un semis de bazars ruraux dans les collines, d'où émergent la petite ville de Pokhara et le bourg de Gorkha, et à une série d'agglomérations qui ont fleuri depuis les années cinquante dans la plaine du Téraï, le long de la frontière indienne. Jusque dans les années soixante-dix, le Népal ne disposait d'aucun système de transport digne de ce nom permettant un accès commode aux principales agglomérations du pays et a fortiori aux villages de montagne. En 1955, le réseau carrossable se réduisait à $624 \mathrm{~km}$ de pistes de mauvaise qualité. L'aide financière de l'Inde et de la Chine a permis la construction d'une demi-douzaine de routes carrossables, soit $7500 \mathrm{~km}$, mais les meilleures équipent la plaine du Téraï. Celles qui traversent les collines et montagnes du Népal sont encore peu nombreuses et fréquemment éboulées pendant la mousson. Une seule route goudronnée, qui mène de Kathmandou à Lhasa, permet de franchir la chaîne himalayenne au niveau du poste frontière de Kodari à $1700 \mathrm{~m}$ d'altitude. Un certain nombre d'altiports ont aussi été construits pour relier à la capitale les zones les plus reculées du pays. Mais leur trafic est fortement contraint par les conditions atmosphériques, et limité par la taille des appareils (figure 1).

6 Dans ces conditions, les déplacements en montagne ne peuvent s'effectuer qu'à pied et le transport des marchandises à dos d'homme ou d'animal, mais l'utilisation d'animaux de bât n'est possible que sur des itinéraires limités. Paysans, bergers, marchands et pèlerins se déplacent donc à pied sur de longues distances et font du portage depuis toujours. En l'absence de routes carrossables, les paysanneries himalayennes ont construit un réseau extrêmement dense de sentiers hiérarchisés. Ils sont les vecteurs d'une vie commerciale intense qui reste indispensable à l'économie du pays, malgré les contraintes opposées par le relief.

Les grands chemins du commerce caravanier transhimalayen

7 Le Moyen-Pays népalais, qui couvre toute la partie centrale du pays, présente un volume montagneux très compact qui n'offre que peu d'aération. La chaîne 
himalayenne est coupée par quelques grandes vallées perpendiculaires, mais celles-ci présentent un profil en "V" très accusé, accidenté de gorges, où l'espace entre le lit du torrent et la falaise est très réduit. Les sentiers qui s'accrochent à leurs parois sont souvent difficiles et dangereux pour les villageois qui les parcourent en transportant sur leur dos des charges lourdes et volumineuses. Ces profondes vallées, qui s'élargissent plus au nord en de grandes auges glaciaires, sont les seules voies de communication et d'échanges avec le Tibet voisin accessibles aux animaux de bât. Des convois de mules, ornées de pompons et de carillons, y transportent depuis des siècles le grain et le sel sur les chemins escarpés qui relient le Népal au le Tibet. La vallée de la Kali Gandaki qui se faufile entre le massif du Dhaulagiri et celui de l'Annapurna, et, dans une moindre mesure, celle de la Marsyangdi un peu plus à l'est, sont les principales voies de ce commerce transfrontalier dans le centre-ouest du Népal. Il est monopolisé par les Thakali et les Manangi, populations d'origine tibétaine, vivant dans la partie supérieure de ces vallées. Cette tradition commerçante leur a permis de s'ouvrir largement vers le monde extérieur : leurs voyages les ont conduits dans toute l'Asie du sud-est et jusqu'en Europe. Enrichis par le commerce, ils se sont installés en nombre à Kathmandou.

8 A haute altitude, les mules sont remplacées pour le passage de la frontière par des yacks, mieux adaptés au froid et à la neige. La vallée glaciaire de la Dudh Kosi, rivière qui prend sa source dans le massif de l'Everest, est une grande voie caravanière pour les yacks qui traversent la frontière par le col du Nangpa-la à $5716 \mathrm{~m}$ d'altitude. Elle relie Namche Bazar situé à $3500 \mathrm{~m}$ d'altitude à Tingri au Tibet, bourgade reliée aux grands itinéraires de la route de la soie. Le commerce est le seul moyen de compléter des ressources agricoles insuffisantes dans ce milieu ingrat de haute altitude. Il a longtemps été la principale source de revenus pour les Sherpa du Khumbu, ethnie d'origine tibétaine qui vit au pied de l'Everest. Sel, animaux, couvertures de laine, plantes médicinales et céréales sont les principaux biens échangés.

Il existe une trentaine d'autres cols d'altitude entre $5000 \mathrm{~m}$ et $5800 \mathrm{~m}$ qui permettent la communication et le commerce du sel, des grains, du bétail et d'autres produits locaux entre le Népal et le haut plateau tibétain. Ils sont marqués par des cairns ornés d'exvoto et de drapeaux à prières qui rappellent la culture bouddhiste des populations des hautes terres himalayennes. Cependant, la fermeture de la frontière tibétaine par les autorités chinoises en 1959 a quelque peu ralenti le trafic transhimalayen. Les grandes caravanes partant durant plusieurs mois vers le Tibet ne se pratiquent plus guère et les échanges se font de proche en proche. De nouveaux produits, comme le beurre, les oranges ou les tissus de coton, en provenance des basses vallées, se substituent de plus en plus au sel qui était jusque-là le commerce le plus lucratif (cf. cartons 1 et 2, figure 3). La plupart des grands circuits commerciaux se sont réorientés vers le sud du pays, et les échanges commerciaux à l'intérieur des frontières du Népal profitent aujourd'hui pleinement aux Newar, une ethnie tibéto-birmane qui se concentre dans les bazars des collines du Moyen-Pays. Cette population, sans doute une des plus anciennes du Népal, a joué un rôle historique fondamental en édifiant des royaumes prospères dans la vallée de Kathmandou, dont l'architecture porte le témoignage. La richesse des anciens royaumes newar reposait autrefois sur la maîtrise du commerce transhimalayen entre le Tibet et l'Inde qui passait par Kathmandou jusqu'au XVIII ${ }^{e}$ siècle. Aujourd'hui les Newar détiennent toujours un quasi-monopole du commerce dans la vallée et contrôlent l'essentiel des déplacements commerciaux entre les bazars du Moyen-Pays, pour lesquels ils emploient des porteurs à gages. Ces bazars sont, comme dans le passé, 
les nœuds d'un commerce actif à l'échelle nationale, utilisant un réseau dense de sentiers, animés par une intense circulation (figure 2).

Le portage commercial à dos d'homme : une activité économique vitale

L'essentiel du portage est lié au commerce et fait partie des migrations saisonnières de travail qu'effectuent les montagnards pendant la morte-saison agricole. Celles-ci ne durent qu'un ou deux mois en général et s'exercent à l'échelle locale ou régionale, nourrissant d'importants échanges de denrées ou de produits manufacturés entre le haut et le bas-pays. Les marchandises les plus variées sont transportées à dos d'homme : bois et fourrage, produits alimentaires (grains et farines, fruits, pommes de terre, nouilles chinoises, épices, thé, sel indien ou tibétain, sucre, huile, bouteilles de bière et de boissons gazeuses), matériaux de construction (planches et poutres, tôle ondulée et câbles en acier, sable et ciment), bidons de kérosène, laines et tissus, plantes médicinales, produits manufacturés d'usage courant (allumettes et cigarettes, savon, piles, bougies, ustensiles de cuisine), bijoux et objets de culte tibétains, ou encore montres et transistors achetés à Hongkong, Singapour ou Brunei. Le transport de ces marchandises s'effectue généralement dans de grandes hottes en vannerie à bandeau frontal, ou avec une simple courroie, maintenant sur le dos les sacs de grains ou les ballots de tissus.

11 Le portage s'insère dans la pluriactivité montagnarde, commune à la plupart des agriculteurs du Népal. Il faut cependant distinguer le portage pratiqué en compte propre par des paysans et qui s'effectue dans le cadre de l'économie agricole au sein de la famille, du portage salarié qui s'effectue pour le compte d'un paysan riche, d'un commerçant newar ou d'une entreprise de travaux publics.

Le premier type de portage s'effectue à l'échelle locale par les paysans qui vont vendre leurs produits agricoles au bazar ou dans les villages voisins et acheter des biens de consommation courante. Certains d'entre eux se lancent cependant dans des transactions plus complexes en pratiquant le portage commercial pour leur propre compte, à l'échelle régionale. C'est ce qu'on appelle le système bepari : il faut pour cela posséder des surplus au village ou avoir une mise de fond personnelle qui permet d'acheter au bazar le plus proche des marchandises à échanger dans des vallées où ces produits ne sont pas disponibles. De bazar en bazar, le prix des marchandises s'accroît le long des vallées où ce type de commerce est pratiqué. Chaque vallée, chaque village possède ses propres filières d'acheminement de ces marchandises. Le bénéfice du marchand-bepari tourne autour de $30 \mathrm{NR}$ par jour ${ }^{1}$. Mais s'il doit l'emprunter, il est rogné par les intérêts de son emprunt. D'une façon générale, les risques sont importants pour des profits médiocres.

13 Par ailleurs, les besoins de déplacements et de transport de marchandises ou de matériaux de construction à l'échelle régionale ont donné lieu à une véritable activité professionnelle salariée qui occupe de nombreux paysans pendant la morte-saison. Pour assurer le transport des marchandises générales à l'intérieur du pays et vers la frontière indienne, le gouvernement népalais appointait jusque dans les années cinquante des hommes, les naiké, chargés de recruter des porteurs à gages dans les villages. A l'époque, ces naiké étaient payés 5 NR à 6 NR par jour pour transporter une trentaine de kilos pendant les douze jours de marche nécessaires pour joindre Kathmandou à Pokhara.

14 Aujourd'hui, ce travail salarié s'exerce la plupart du temps dans un cadre privé : les paysans riches ou les boutiquiers de village emploient périodiquement des porteurs à 
gages pour effectuer les tâches de transport à leur place. Ils les payent environ $400 \mathrm{NR}$ sans la nourriture pour transporter $50 \mathrm{~kg}$ de marchandises sur cinq jours de marche environ, soit $80 \mathrm{NR}$ par jour. Sur le chemin, le porteur doit dépenser pour sa nourriture et son logement environ $250 \mathrm{NR}$. Il ne lui reste donc plus que $150 \mathrm{NR}$ au terme de son aller-retour, soit un salaire de $30 \mathrm{NR}$ par jour. C'est pourquoi ces porteurs emportent souvent avec eux de la farine de maïs, du soja ou du millet grillé qu'ils peuvent consommer en cours de route.

preurs à gages professionnels qui travaillent pour un marchand ou une entreprise à l'extérieur de leur village sont payés de même au poids et à la distance parcourue, calculée en jours de marche. Leur salaire varie entre 8 et 15 NR par jour. La charge transportée peut atteindre 80 à $100 \mathrm{~kg}$. Ce type de portage peut s'exercer dans des régions fort éloignées du village et sur des trajets assez longs. Les gains sont alors plus élevés. Mais quels que soient les écarts de salaires que l'on enregistre ici et là, les activités de portage ne procurent que de faibles revenus et toujours pour un travail pénible. Cependant ces médiocres salaires sont souvent le seul moyen de survie des paysans pauvres que l'exiguïté de leur terre ne permet pas d'occuper toute l'année.

Le portage à gages ne concerne pas que les marchandises : des personnes en voyage peuvent aussi engager des hommes pour porter leurs affaires. Autrefois, le portage était souvent effectué comme travail gratuit pour le compte d'un chef de village, tandis que les souverains locaux ou les gouverneurs de province utilisaient officiellement des coolies pour transporter leurs impedimenta au cours de leurs déplacements. Aujourd'hui, des porteurs professionnels louent fréquemment leurs services à des soldats permissionnaires de retour dans leur village après leurs trois années d'engagement à l'étranger, à des fonctionnaires regagnant leur poste dans les collines ou à de riches citadins népalais qui se déplacent avec leurs bagages pour assister à une fête dans leur village d'origine.

Le réseau des sentiers : un paysage construit et entretenu

Malgré son apparente simplicité, le transport à dos d'homme requiert d'importants aménagements. Chaque village construit et entretient collectivement son réseau de sentiers qui escaladent les versants cultivés et de larges portions de forêt, en d'impressionnantes volées de marches d'escalier en pierre : elles permettent le passage des hommes chargés de hottes de foin ou de bois mais aussi celui des animaux lors de leurs transhumances vers les pâturages d'altitude. La circulation sur les versants enterrassés se fait le long des canaux d'irrigation et des murets qui soutiennent les champs. De grands chemins à flanc de montagne, les mul bato, relient les villages entre eux. C'est en pays gurung au pied du massif de l'Annapurna, que ces aménagements sont les plus remarquables et les mieux entretenus, car cette ethnie montagnarde exploite de façon systématique des terroirs qui s'étendent sur plusieurs étages bioclimatiques: des champs en terrasses de l'étage subtropical jusqu'aux pelouses de l'étage alpin. La construction et la réfection de ces infrastructures fait partie des contraintes collectives acceptées par toutes les communautés montagnardes.

Les vallées himalayennes sont la plupart du temps très encaissées et n'offrent que peu de points de franchissement: les ponts consistent souvent en une simple passerelle formée d'un tronc d'arbre ou de deux poutres sur lesquelles sont posées des planches ou des pierres plates. On rencontre encore fréquemment des ponts suspendus en lianes et bambous, pour les plus rudimentaires, ou solidement arrimés par des câbles d'acier et aménagés avec des planches clouées, ou maintenues par des dalles de pierre pour les 
plus récents. Ces derniers sont construits avec l'aide du gouvernement et peuvent être empruntés par des animaux, chèvres, vaches, mules et même yacks. Certaines rivières, où un véritable pont ne peut être construit, sont équipées d'une nacelle suspendue à un filin. La traversée se fait par gravité et à l'aide d'une corde tractée par le passager ou par un passeur installé sur l'autre rive.

Le long des gorges des rivières, les sentiers sont parfois taillés dans la roche en place et consolidés par quelques madriers et treillis garnis de cailloux lorsque le passage surplombe les torrents. En mousson, ces chemins sont particulièrement glissants et peuvent provoquer des chutes mortelles pour les hommes comme pour les animaux; c'est pourquoi la vie de relation à l'échelle locale s'effectue le plus souvent entre des villages situés de part et d'autre des lignes de crêtes.

Tout au long des chemins, sont aménagés à intervalle régulier des cautara, longues murettes de pierres construites sous de grands arbres, les pipal et les bar qui apportent l'ombre aux heures chaudes de la journée. Les porteurs peuvent, sans quitter leur charge, y appuyer leur doko, lourd panier d'osier ou de bambou maintenu par un large bandeau de cuir passé autour de leur front. Sans ces arrêts-reposoirs, le transport de marchandises à dos d'hommes serait difficilement praticable, car la hotte ne tient debout, une fois équilibrée, qu'appuyée contre un support. Ces cautara se rencontrent de façon systématique dans les collines du centre-ouest du Népal jusqu'à la limite de la forêt. Dans l'est du Népal, et sur les chemins de crête, les cautara sont plus rares. Les porteurs raï, ethnie qui vit dans les collines des districts de l'est du Népal, ont une façon originale de se reposer : ils appuient la pointe de leur panier sur de longs bâtons à la poignée en forme de "T" qu'ils utilisent pendant leur marche.

21 Lorsque les transports durent plusieurs jours, les porteurs campent le long du sentier dans des abris sous roche ou dans les dharamsala, abris rudimentaires pour pèlerins aménagés à l'entrée de certaines agglomérations. Ils mangent la nourriture qu'ils ont apportée de leur village ou bien se nourrissent et se logent pour la nuit dans des auberges modestes appelées bhatti. Ce sont des lieux très importants de la vie sociale car c'est là que les gens échangent les nouvelles et c'est par les porteurs que circule l'information en l'absence de téléphone et de journaux. Les conversations portent aussi bien sur l'état des chemins et des ponts ou les conditions atmosphériques, que sur les nouvelles concernant des proches, mariages, décès, sur le prix du grain dans les villages avoisinants, les offres d'emplois saisonniers ou les derniers événements survenus dans la capitale ou dans le bourg le plus proche.

Du portage commercial au portage pour le trekking

Depuis une trentaine d'année, le portage salarié s'est ouvert à un nouveau marché, celui du tourisme de trekking. Ce terme désigne une randonnée pédestre de plusieurs jours dans un massif montagneux extra-européen. Le Népal constitue le modèle pionnier de ce tourisme qui s'est développé à partir des années soixante sur les traces des premières grandes expéditions himalayennes. Il repose sur l'attraction que représentent les plus hautes montagnes de la terre, sur l'existence de cet important réseau de sentiers, et surtout sur la disponibilité d'une main-d'œuvre nombreuse et bon marché, rompue à la mobilité et la pénibilité du portage salarié: les touristes qui pratiquent le trekking dans l'Himalaya sont encadrés par une équipe locale de guides, de cuisiniers et de porteurs, chargée d'acheminer à dos d'homme les sacs des randonneurs, la nourriture et le matériel de camping². 

commercial traditionnel. Il n'a eu qu'à reproduire les modes de fonctionnement auxquels les paysanneries népalaises étaient soumises depuis longtemps dans leurs migrations temporaires de travail. De plus, le trekking a pu se couler sans peine dans ce réseau de sentiers constamment entretenu par les villageois et dont il ne reprend qu'une infime partie des itinéraires. Ils correspondent en général à ceux qui ont été empruntés par les premières expéditions himalayennes en direction de l'Everest et de l'Annapurna, pour les circuits les plus fréquentés, et à certains tronçons des chemins caravaniers (haute Kali Gandaki, Bhuri Gandaki) - cf. figures 2 et 4.

L'organisation primitive du portage pour le compte des expéditions himalayennes

A l'époque pionnière de l'exploration himalayenne et de la conquête des grands sommets, les alpinistes devaient franchir des distances démesurées et réaliser de pénibles marches d'approche pour atteindre le camp de base des montagnes, depuis la jungle infestée de malaria jusqu'aux hautes vallées se succédant interminablement derrière chaque ligne de crête. Tout cela dans des régions pratiquement inconnues, sans cartes dignes de ce nom. Le recrutement de guides locaux connaissant les langues régionales et capables de négocier avec les différentes ethnies rencontrées, ainsi que l'utilisation de centaines de porteurs pour acheminer des semaines de vivres et tout le matériel d'escalade et de protection contre le froid, étaient une condition préalable à l'organisation de telles entreprises. Ainsi, lorsque les premières expéditions ont recherché des guides et des porteurs, elles n'ont eu aucun mal à embaucher une maind'œuvre paysanne, habituée aux travaux de force, et toujours à l'affût d'un appoint monétaire qui leur permette de survivre sur des terres aux ressources précaires.

Avant 1950, toutes les expéditions qui se portaient sur l'Everest opéraient depuis la station climatique de Darjeeling, au Bengale, car le Népal était alors interdit aux étrangers. C'est là que furent recrutés les premiers porteurs parmi les migrants temporaires qui venaient chercher du travail dans les plantations de l'Assam ou les chantiers de construction de l'empire britannique. La plupart venaient du Moyen-Pays népalais sous la conduite d'un de leurs "frères de clan $»^{3}$ qui jouait le rôle de naiké, c'est-à-dire d'intermédiaire avec leur futur employeur. Parmi eux, se trouvaient aussi de nombreux Sherpa, venus du Khumbu, qui s'illustrèrent dans la conquête de l'Everest et devinrent rapidement les auxiliaires principaux des alpinistes et les chefs des équipes de portage. Dès 1927, les alpinistes britanniques fondèrent l'Himalayan Club, dont la section de Darjeeling était la plus organisée. Ils instaurèrent des règles, imitées de l'Alpine Club, en Europe, mais aussi des traditions locales concernant le recrutement, les salaires et l'équipement des porteurs locaux. Les naiké qui travaillaient préalablement pour le recrutement des porteurs de marchandises furent chargés de les diriger sur les expéditions, organisant de véritables filières d'emplois qui perdurent toujours. C'est ainsi que Jit Bahadur "Naïké", un Tamang ${ }^{4}$ du village de Temal Parsel à l'est de la vallée de Kathmandou, engagea 800 hommes de son village pour accompagner l'expédition de Hunt et Hillary à l'Everest en 1953. De là date une véritable vocation de portage dans ce village où aujourd'hui près des trois quarts des hommes travaillent dans le trekking ou l'himalayisme.

Lorsque le Népal a enfin ouvert ses frontières aux occidentaux, le trekking a pu se développer à partir de Kathmandou, où s'étaient créées des agences de trekking qui n'ont eu qu'à reprendre les modes de recrutement prévalant pour les expéditions. 
Depuis s'est imposée la terminologie toujours en vigueur dans le trekking, où il faut distinguer l'équipe népalaise d'encadrement des touristes, des simples porteurs.

Le sardar est le chef de l'équipe locale, recrutée pour une expédition ou un trek. Il appartient très souvent à l'ethnie sherpa. Il est chargé du recrutement de ses hommes de confiance qu'il choisit presque toujours parmi ses frères de clan. Ses responsabilités le place au sommet de la hiérarchie du trekking, avec des salaires de l'ordre de $200 \mathrm{NR}$ par jour. Certains travaillent de façon permanente pour la même agence de trekking et peuvent alors obtenir leur mensualisation, ce qui les met à l'abri des irrégularités de cette activité saisonnière.

Le terme de sherpa dans les métiers du trekking a un sens professionnel qu'il ne faut pas confondre avec son sens ethnique qui désigne les seuls habitants du Khumbu: les sherpas, qui travaillent sous les ordres du sardar, sont employés pour monter les tentes, et surveiller la bonne marche du trek. Dans les expéditions, les sherpas d'altitude, appelés encore "porteurs de haute altitude", ou parfois climbing sherpa pour ceux qui possèdent des techniques d'escalade, sont chargés d'acheminer le matériel d'ascension, de poser les cordes fixes ou des échelles pour le franchissement des crevasses, et d'installer les camps d'altitude. Ils ont donc des tâches de portage, mais à un niveau de qualification bien supérieur à celui d'un simple coolie, dans la mesure où ils doivent aussi assister la progression des alpinistes. Ils reçoivent entre 100 et 150 NR par jour.

Vient ensuite le personnel de cuisine : toute expédition ou trek organisé s'adjoint un cuisinier, le cook, et deux ou trois aides, les kitchen-boys, qui servent d'hommes à tout faire : vaisselle, coupe du bois et autres corvées, dont les salaires sont assez proches de ceux des sherpa.

Quant aux porteurs proprement dit ou coolies, ils portent en théorie des charges de 30 $\mathrm{kg}$, mais souvent beaucoup plus, pour moins de $100 \mathrm{NR}$ par jour. Contrairement au reste de l'équipe népalaise, ils ne sont ni nourris, ni logés durant le trek, ce qui réduit d'autant leurs gains. Leur tâche et leur statut ne sont guère différents de ceux des porteurs des collines travaillant pour un marchand newar, bien que leur salaire soit en général un peu plus élevé, grâce aux pourboires que leur versent les touristes. Ce sont des saisonniers recrutés soit par un naiké, soit directement par le sardar, ou qui se sont présentés spontanément à l'embauche, en faisant le tour des bureaux des agences de trekking de Kathmandou, ou en attendant près des gares routières ou des altiports par lesquels arrivent les groupes. Ils viennent souvent du même village par groupes de trois ou quatre, et appartiennent soit à la même famille, soit au même clan.

La fonction du naiké enfin, subsiste toujours sous une forme assez similaire à celle du portage commercial : le naiké en trekking est le chef du groupe de porteurs et leur porte-parole auprès du sardar. Il les accompagne au cours du voyage et négocie pour son groupe les conditions d'emploi. En contact avec les agences de trekking, il peut servir aussi d'agent recruteur dans un groupe de villages et se paie en prélevant luimême sa commission sur le salaire de chaque porteur à qui il a trouvé un travail. Il existe par ailleurs aujourd'hui des naiké salariés d'agences de trekking. Installés dans les bazars situés au pied des routes de trek, ils reçoivent un salaire de l'agence qui les emploie et ne participent pas au trekking.

Les porteurs de trekking : une nouvelle dynamique socio-spatiale

32 Le portage de trekking et d'expédition apparaît en de nombreux points comparable au portage à gages des marchandises, mais malgré la modestie et l'irrégularité des revenus qu'il procure, il est nettement plus avantageux que les travaux saisonniers équivalents 
qui sont accessibles aux villageois sans qualification. Le travail dans le trekking, outre qu'il est légèrement mieux payé, a un caractère un peu moins pénible : les charges sont moins lourdes, bien que transportées plus longtemps et souvent dans des conditions difficiles (passages de cols sous la neige par exemple). Mais les porteurs ne sont pas isolés quand ils abordent des passages pénibles ou dangereux car ils marchent ensemble, souvent entre frères de clan, en s'encourageant mutuellement. Ils jouissent malgré tout d'une considération un peu meilleure de la part des étrangers que de la part des marchands newar qui les emploient d'ordinaire dans le portage commercial. Mais surtout le portage pour les touristes offre aux plus motivés des possibilités de progression vers d'autres tâches mieux rémunérées. Au bout de quelques saisons, un porteur ou un kitchen-boy peut devenir sherpa, puis guide ou cuisinier: sa situation matérielle peut alors s'améliorer assez nettement par rapport à celle qu'il aurait en pratiquant des tâches de simple homme de peine. Même si les revenus d'un guide de trekking sont très inégaux selon le nombre de treks qu'il peut réaliser dans l'année, ils sont toujours bien supérieurs à toutes les activités de portage ou autres petits métiers d'appoint que peuvent exercer les villageois. Seul le mercenariat pour le compte de l'armée britannique ou indienne offre des revenus et un statut comparables à ceux du trekking. Mais l'enrôlement dans les garnisons britanniques d'Asie du Sud-Est, qui a longtemps été massif auprès des montagnards du Moyen-Pays, se tarit progressivement.

Les régions montagneuses du Moyen-Pays, qui sont aujourd'hui les grands bassins de main-d'œuvre pour le trekking, supportent de fortes densités de population sur des terres trop peu productives. Elles ne peuvent conserver leurs structures économiques et sociales qu'au prix de migrations temporaires ou définitives qui agissent comme une soupape de sécurité en soulageant la pression démographique sur les terres. Le portage commercial pratiqué dans le cadre de la pluriactivité montagnarde avait jusqu'à présent permis de pérenniser la situation économique de ces sociétés paysannes enracinées dans leur terroir. Il ne constituait qu'un complément de ressources pour les exploitations agricoles dont il permettait la survie et le moyen le plus simple pour s'acquitter d'une dette ou acquérir des revenus monétaires indispensables aux échanges marchands.

Le succès des emplois dans le trekking auprès des jeunes villageois népalais s'inscrit donc dans le contexte d'une économie montagnarde qui ne suffit plus à nourrir les hommes. Alors que les migrations temporaires de travail traditionnelles ne remettaient pas fondamentalement en cause les structures sociales et l'organisation de l'espace, le tourisme de montagne introduit par sa propre dynamique et ses propres flux migratoires des effets non négligeables sur ces sociétés montagnardes, mais aussi sur la structuration du territoire népalais.

Tout d'abord, les revenus plus substantiels qu'offre le trekking à ceux qui ont su se hisser au rang de sardar, l'instruction acquise sur le tas au contact des touristes et les nouvelles mentalités plus individualistes qu'entraîne le travail salarié, offrent à ces derniers l'opportunité de conquérir une certaine indépendance matérielle, qui les éloigne de plus en plus des contraintes de la vie agricole. Pendant un temps, le porteur rapporte la plus grande partie de ses gains au village, mais peu à peu ses visites se raréfient, car le porteur préfère enchaîner plusieurs treks à la suite et rester à Kathmandou le temps de trouver un nouvel engagement. 

spectaculaires, les plus exceptionnellement signifiants, le trekking contribue à différencier les massifs montagneux du Népal: ce sont ceux qui sont attachés à la présence d'une montagne célèbre par sa hauteur ou les ascensions dont elle a fait l'objet, comme l'Everest ou l'Annapurna, ou dont la forme est particulièrement repérable. Là où les autres sommets ne sont connus que d'un cercle limité d'amateurs de montagne et d'alpinisme, c'est la présence de populations ou de monuments remarquables représentatifs de la culture la plus signifiante du Népal pour le touriste, qui servent de marqueurs territoriaux : ainsi, les régions où s'observent les signes de la culture bouddhiste sont survalorisées par le tourisme. L'association montagne himalayenne et hindouisme est moins répandue. Beaucoup de touristes ne réalisent que sur place que l'hindouisme est la religion majoritaire au Népal. Cependant, quelques sites correspondant à des lieux de pèlerinage hindous, comme le sanctuaire de Muktinath dans la région de l'Annapurna ou les lacs sacrés de Gosainkund dans le Langtang, sont désignés comme le point d'apogée de certains itinéraires.

41 A cette "mise en site" par le tourisme des massifs montagneux s'ajoute une offre locale qui permet leur fréquentation, et un contrôle institutionnel qui organise de plus en plus les flux. Le gouvernement népalais n'autorise pas la libre circulation des 
étrangers dans l'ensemble du pays. Pour réaliser une randonnée en montagne, les touristes doivent se munir d'un permis payant délivré par l'Office de l'Immigration. Leur coût varie selon le mode d'organisation de la randonnée et selon la région. Pour les circuits classiques, ils tournent autour de quelques dollars par semaine. Les itinéraires autorisés sont spécifiés sur le permis de trekking qui n'est valable que pour une région à la fois et pour un nombre de jours déterminé. Sur les itinéraires de trekking, des fonctionnaires de police, installés dans des check post placés à l'entrée des vallées principales et dans les villages donnant accès aux cols, sont chargés de vérifier les permis et de pénaliser les contrevenants. La surveillance est d'autant plus sévère que de nombreuses régions sont fermées aux étrangers, en particulier celles qui bordent la frontière tibétaine considérée comme zone stratégique (cf. figure 4).

La fréquentation des massifs se distingue aussi en fonction des différents types de pratiques touristiques. Une fraction importante de la population des trekkeurs pratique la randonnée en individuel en compagnie d'un simple porteur, en utilisant les ressources locales en logement et nourriture qui n'existent que sur un petit nombre d'itinéraires : massifs de l'Annapurna, de l'Everest et du Langtang. Les autres touristes passent par les services d'une agence de trekking qui leur fournit une équipe complète de porteurs, de sherpas et de cuisiniers. Ils ne sont donc pas dépendants des structures d'hébergement.

43 L'Annapurna, l'Everest et le Langtang sont les régions les plus visitées par les trekkeurs. En 1991, le massif de l'Annapurna a concentré à lui seul 16875 randonneurs, et celui de l'Everest 7990, tandis que la région du Langtang et d'Helambu recevait 4789 visiteurs. La fréquentation confondue des autres régions du Népal ouvertes au trekking ne concerne quant à elle que 3495 visiteurs. Trekkeurs individuels et groupes organisés se partageaient à peu près également sur le trek de l'Everest; les premiers étaient environ 3000 de plus que les seconds sur le tour de l'Annapurna, alors qu'ils étaient près de trois fois plus nombreux dans le massif du Langtang. Ce sont aussi les touristes individuels qui assurent l'essentiel de la fréquentation des massifs en dehors des deux périodes de haute saison de l'automne et du printemps, alors que les groupes organisés se concentrent sur ces périodes les plus favorables à la pratique du trekking. Ces différences s'expliquent par la durée, le niveau de difficulté, l'accessibilité des massifs et la qualité des équipements rencontrés. Le Langtang est, par sa proximité de Kathmandou, ses altitudes moyennes peu élevées et le nombre d'auberges installées sur le chemin, la région qui se prête le mieux aux randonnées que l'on organise soi-même. Le massif de l'Everest est le moins facile d'accès : seul un altiport accueillant de petits twin-otters de 15 places permet d'éviter la longue marche d'approche qui conduit au Khumbu. De plus, l'itinéraire qui mène au camp de base de l'Everest se déroule entre $3500 \mathrm{~m}$ et $5200 \mathrm{~m}$ d'altitude. En revanche, il est très bien équipé en auberges qui évitent aux touristes de camper même à haute altitude. Le massif de l'Annapurna, quant à lui, est facilement relié à Kathmandou par la route. Pokhara, point de départ ou d'arrivée des nombreux circuits de la région, possède aussi un aéroport qui la relie aussi bien à la capitale qu'à l'altiport de Jomossom, dans la haute Kali Gandaki. Seul le tour complet du massif oblige les randonneurs à franchir un col de plus de $5000 \mathrm{~m}$ d'altitude.

Depuis quelques années, de nouveaux massifs ont été ouverts au trekking. Le gouvernement népalais s'est rendu compte qu'il risquait de perdre au profit de la Chine une fraction non négligeable de touristes, intéressés par la découverte des régions de culture tibétaine. Or le Népal peut se prévaloir de posséder sur son territoire quelques 
régions aux paysages comparables à ceux du Tibet: leur population a pu conserver l'essentiel de ses traditions culturelles ancestrales, ce qui n'est malheureusement plus le cas de l'autre côté de la frontière, où les monastères bouddhistes ont beaucoup souffert des persécutions du gouvernement chinois. C'est la raison pour laquelle le gouvernement népalais a autorisé progressivement les trekkeurs à visiter le Dolpo, le Manaslu, le Kanchenjunga et le Mustang, mais à la condition qu'ils passent par les services d'une agence de trekking homologuée et soient accompagnés d'un fonctionnaire de police. Ces nouvelles régions ouvertes aux randonneurs se caractérisent toutes par la rareté des ressources et par des équilibres naturels extrêmement fragiles. C'est pourquoi les groupes de trekkeurs doivent être totalement autosuffisants en nourriture et en combustible (gaz ou kérosène), d'où l'obligation de passer par une agence de trekking. Ces restricted areas font l'objet d'une tarification spéciale : 10 et $15 \$$ par semaine pour le Dolpo et le Kanchenjunga, $75 \$$ pour le tour du Manaslu et 500 \$ pour le Mustang. Le gouvernement justifie ces tarifs élevés par la volonté de limiter la fréquentation touristique de ces régions pour protéger le milieu naturel et les populations. Il ne réserve de ce fait l'accès à ces régions qu'à un petit nombre de personnes à hauts revenus voyageant en groupes, qu'il peut ainsi plus facilement contrôler. On comptait en 1991 pour ce type de circuits 2659 trekkeurs : 563 se sont dirigés vers le Kanchenjunga, 500 vers le Dolpo et 168 seulement vers le Mustang et le Manaslu, le reste se dispersant sur des itinéraires non réglementés et moins connus.

Une certaine différenciation de l'espace s'opère donc en fonction de la nature de la clientèle et de la forme de trekking pratiquée, individuelle ou en groupe, ainsi que de la valeur assignée par le montant des permis. Ainsi s'opposent les itinéraires classiques fréquentés massivement par les individuels comme par les groupes, et les restricted areas visitées par un petit nombre de groupes qui payent le prix fort pour accéder à des itinéraires inédits. La marque du passage des touristes dans l'espace est elle aussi inégale, puisque les nouvelles régions ouvertes ne possèdent pas de structures d'hébergement, les groupes de randonneurs étant obligés de camper et de transporter toute leur nourriture avec eux depuis Kathmandou.

En revanche, les grands itinéraires classiques sont devenus des axes structurants, engendrant de nouvelles polarisations dans l'espace: de modestes équipements touristiques jalonnent les sentiers en se concentrant sur ces lieux d'étapes dont la localisation n'est pas indifférente. C'est à cette échelle micro-locale que se manifeste un puissant effet de lieu : l'installation d'un site de camping répond à des critères objectifs liés d'abord aux contraintes naturelles: lieu protégé du vent et de la pluie, par exemple, pour installer un coin cuisine et abriter les porteurs qui dorment souvent dehors; présence d'un point d'eau, de combustible et d'un terrain plat où fixer les tentes; proximité d'un village permettant l'approvisionnement en produits frais, tout en conservant un certain éloignement pour préserver à la fois la tranquillité des villageois et celle du groupe souvent sollicité par des enfants quémandeurs. Les campements sont donc souvent aménagés sur des champs en terrasses, en bordure d'un torrent ou parfois dans la cour d'une école de village. Leur choix cherche aussi à répondre autant que faire se peut à d'autres critères exigés par les touristes, comme la qualité des paysages ou la présence de sites remarquables, temples ou monastères que le voyageur désire visiter. 

étapes d'équipements et de services plus ou moins élaborés, lodges, tea-shops, petites épiceries et parfois boutiques de vente de souvenirs. Les lodges sont des auberges rudimentaires qui offrent le gîte et le couvert aux randonneurs. Les tea-shops, souvent installées dans une maison privée, proposent du thé et de la nourriture aux gens de passage et parfois une natte pour la nuit. Ils ont fleuri sur les sentiers de trekking, concurrençant les bhatti fréquentés surtout par les porteurs à gages népalais. Les lodges, qui étaient à l'origine un hébergement chez l'habitant, tendent à s'autonomiser par rapport à l'habitation principale de leur propriétaire. La plupart des lodges qui sont situés sur les plus hautes étapes de trekking ont été aménagés dans des cabanes d'alpage, occupés autrefois de façon temporaire lors de la conduite des troupeaux. Aujourd'hui, grâce au trekking, certains de ces sites d'altitude sont occupés de façon permanente et on y cultive des pommes de terre destinées à l'alimentation des touristes. Les lodges se concentrent inégalement selon les villages. La localisation de l'habitat sur des itinéraires plus ou moins fréquentés et leur espacement par rapport au rythme moyen de marche des trekkeurs en sont les principales raisons. Les villages qui servent d'étape pour la nuit ou les sites remarquables qui constituent les points d'apogée des circuits de trekking ont vu fleurir de nombreux lodges, à l'inverse de ceux qui sont à l'écart des itinéraires ou qui sont seulement traversés au cours de la journée.

the plupart du temps des commerçants newar, ou de riches caravaniers thakali, manangi ou sherpa, ainsi que les propriétaires terriens locaux aisés, qui ont surtout profité du passage des randonneurs. Ils investissent leurs revenus commerciaux dans la construction de lodges et vendent aux touristes leurs surplus agricoles à des prix largement supérieurs à ceux pratiqués d'ordinaire. Ils provoquent de ce fait une forte inflation dans ces régions, qui interdit l'accès à ces surplus des paysans les plus pauvres. Leur réussite par rapport au standard de vie villageois a encouragé des habitants moins fortunés à construire des lodges à leur tour en s'endettant avec l'espoir de rapides amortissements. Dans certains cas, sous l'effet de la concurrence, les prix d'hébergement et de repas n'ont pas suivi le cours de l'inflation et leurs bénéfices sont devenus trop faibles pour permettre de rembourser les dettes et de maintenir un niveau de service acceptable. Le passage des touristes a donc suscité de nouvelles sources de revenus dans ces régions de trekking, mais s'il a provoqué localement l'enrichissement de certains, il a aussi accru les inégalités entre ceux qui avaient déjà les moyens de s'y investir et ceux qui ont continué à pratiquer l'agriculture ou l'élevage traditionnel.

Par ailleurs, de nouveaux trafics de marchandises se sont développés entre les régions de trekking et les bazars du Moyen-Pays pour alimenter les lodges, tea-shops, mais aussi de nombreuses petites échoppes destinées à la consommation des touristes. A côté du thé et du sucre, on achemine maintenant des boissons gazeuses, de la bière, des nouilles chinoises déshydratées en sachet, des boîtes de conserves et de l'épicerie en tout genre, ainsi que des pellicules photographiques, des cartes postales ou des souvenirs. Ces nouveaux produits s'ajoutent à ceux que transportent traditionnellement les porteurs à gages ou les marchands-bepari.

De la même façon, le trekking a suscité sur les chemins de nouveaux besoins en maind'œuvre pour la construction des lodges, le transport du bois destiné au chauffage et à la cuisson des aliments des restaurants, ou pour des tâches de services en rapport avec l'hébergement et la restauration. Les charpentiers et les menuisiers peuvent gagner là 
entre 150 NR à 200 NR par jour moins la nourriture, salaire qui est supérieur à ceux qui sont pratiqués à Kathmandou ou dans les bazars. Le développement du tourisme dans la région de l'Annapurna, de l'Everest et dans une moindre mesure du Langtang, a donc provoqué d'importants courants migratoires en provenance des villages appartenant aux districts voisins.

51 Enfin, la présence des touristes a justifié des équipements nouveaux qu'ignorent la plupart des montagnes du Népal : panneaux solaires, électricité, lignes téléphoniques, altiports, postes de santé, latrines. Des régions montagneuses qui souffraient auparavant de la faiblesse de leurs ressources agricoles et se vidaient périodiquement de leurs populations vers les basses vallées sont devenues des régions riches et attractives. Namche Bazar, petite capitale du Khumbu située à $3500 \mathrm{~m}$ d'altitude au croisement des sentiers qui sillonnent le Parc National de la région de l'Everest, est animée chaque samedi par un marché qui n'existait pas avant les années soixante. Les paysans des basses vallées, pourtant plus favorisées sur le plan agricole, viennent y vendre leurs denrées, attirés par le nouveau pouvoir d'achat des Sherpa du Khumbu. Ces derniers, qui faisaient figure de parents pauvres avant l'irruption du tourisme dans leur région, sont devenus les maîtres enviés d'une activité qui a profondément bouleversé les rapports entre le Haut et le Moyen-Pays.

On voit donc bien que le tourisme de trekking, loin de se plaquer artificiellement sur l'espace et la société locale, s'est inséré dans des infrastructures et des structures économiques préexistantes: le réseau des sentiers du pays a servi de point d'appui à une nouvelle structuration de l'espace qui différencie les régions empruntées par les circuits de trekking de celles qui sont les bassins d'emplois de cette activité ou qui en restent totalement à l'écart. A l'échelle régionale, l'occupation spatiale des montagnes du Népal par le trekking prend une forme linéaire et discontinue : même si les flux monétaires qui s'y diffusent restent modestes, ils ont un impact important sur l'économie locale. Ils permettent le maintien sur place de la population, qui développe, à côté de ses activités agricoles classiques, des activités plus lucratives que celles qui sont engendrées par les courants commerciaux traditionnels. Une économie se substitue à une autre avec comme principal inconvénient d'être très dépendante de l'extérieur. Parallèlement, l'organisation du portage à gages a servi de modèle de fonctionnement au trekking. En offrant des opportunités d'ascension sociale aux paysanneries himalayennes, il concurrence le portage à gages traditionnel dont il est issu, sans pour autant le faire disparaître. En revanche, il détourne du travail de la terre une fraction croissante de ces montagnards et remet en cause des équilibres villageois fragiles. Le Népal ne peut toujours pas se passer du portage salarié, ni d'ailleurs du tourisme de trekking qui contribue pour $44 \%$ aux recettes touristiques du pays, libellées en précieuses devises. Seule l'extension du réseau routier dans les vallées pourrait faire disparaître à terme le portage à dos d'homme. Mais il transformerait aussi profondément la physionomie des régions de trekking en le rendant inutile ou en le cantonnant sur les sentiers des régions les plus hautes et les plus accidentées. C'est alors toute une activité économique et toute une série d'emplois dont il faudrait reconsidérer la fonction et les formes. 


\section{BIBLIOGRAPHIE}

BAUMGARTNER F., BAUMGARTNER R., KOPPE R., ROHRBACH A. (1978), Tourism and Development in Nepal : Impact of Trekking Tourism in hill areas. Report of a field survey in Fall 1977, Zürich, Swiss Federal Institute of Technology, $102 \mathrm{p}$.

DOBREMEZ J-F. dir. (1986), Les collines du Népal central : paysages et sociétés des collines du Népal central, écosystèmes, structures sociales et systèmes agraires. Tome 1 : Paysages et sociétés dans les collines du Népal, tome 2: Milieux et activités dans un village népalais, Paris-Grignon, INRA, $182 \mathrm{p}$.

FISHER J.F. (1990) Sherpa, Reflections on change in Himalayan Nepal, Berkeley, University of California Press.

FÜRER-HAIMENDORF C. (1975), Himalayan Traders. Life in Highland Nepal, London, John Murray, $316 \mathrm{p}$.

GABORIEAU M., SAGANT P., TOFFIN G. (1978), « Les migrations en Asie du Sud », L'Ethnographie, revue de la Société française d'ethnographie, 77-78, $n^{\circ}$ spécial, Paris.

MESSERSCHMIDT D. (1974,) « Parallel trade and innovation in Central Nepal : the cases of the Gurung and Thakali subbas compared ", Contribution to the anthropology of Nepal, London, pp. 197-221.

MÜLLER B. (1990), « Les migrations de travail dans les collines du Népal proche-oriental », Les Cahiers de sociologie économique et culturelle, 13, pp. 151-177.

SACAREAU I. (1995), « Un cas de tourisme diffus à l'échelle d'un pays du Tiers-monde : le trekking au Népal », Le tourisme diffus, Claude JAMOT et Pierre VITTE (dir.), CERAMAC 8, Actes du Colloque de Clermont-Ferrand, Journées de la Commission de géographie du tourisme et des loisirs, 12-14 sept. 1994, Université Blaise Pascal, pp.101-117.

SACAREAU I. (1997), Porteurs de l'Himalaya, géographie du trekking au Népal, Paris, Belin, collection Mappemonde, $260 \mathrm{p}$.

STEINMANN B. (1985), Les porteurs et le Tamba : quelques aspects de la vie ethnique et de sa décomposition chez les Tamang de l'est (Népal), Thèse de doctorat, Université Paris X-Nanterre, 658 p.

VERLIAT S., Villages et maisons dans un espace rural népalais pluriethnique : vallée de la Tamba Kosi, Thèse de doctorat, Université de Bordeaux III, non datée (entre 1988 et 1990), 152 p.

ZURIK D. N. (1992), « Adventure travel and sustainable tourism in the peripherical economy of Nepal », Annals of the Association of American Geographers, vol. 82, $\mathrm{n}^{\circ} 4$.

\section{NOTES}

1. 1 franc français $=10$ roupies népalaises environ

2. Avec plus de 60000 randonneurs et alpinistes par an, les besoins en main-d'œuvre du trekking s'élèvent à environ 35000 emplois.

3. Les ethnies montagnardes tibétaines et tibéto-birmanes du Népal sont divisées en clans exogames qui constituent d'importants réseaux d'alliances à travers les règles de mariage et d'échanges de services.

4. Les Tamang sont une ethnie tibéto-birmane du Moyen-Pays népalais qui sont engagés massivement dans les activités de portage. 


\section{RÉSUMÉS}

En l'absence d'infrastructures de transport suffisantes, une grande partie de la vie de relation au Népal s'effectue grâce à un dense réseau de sentiers. Dans les montagnes du pays qui couvrent les deux tiers du territoire, les marchandises se transportent toujours à dos d'hommes, donnant naissance à une activité salariée de portage à gage, qui s'inscrit dans la pluriactivité montagnarde. Cependant, depuis une trentaine d'années, le tourisme de trekking a superposé ses propres itinéraires au réseau des chemins et offert de nouveaux emplois de portage aux paysanneries himalayennes. La dynamique économique et sociale induite par cette activité contribue à une nouvelle structuration de l'espace à l'échelle du pays.

\section{Trekking tracks ans carriers's activity in Nepal}

Due to the lack of adequate transport infrastructure, a large part of everyday life relations in Nepal exists thanks to a dense network of tracks. Across the country's mountains, covering twothird of its territory, the goods are always carried on people's shoulders. That kind of transportation gave the mountaineers' plurality an extra-job, the wage carriage. Nevertheless, for about thirty years now, the trekking jointed its own tracks to that network and opened new carrier jobs for the Himalayan countryman. The economical and social thrust generated by this activity defined a new space structure countrywide.

\section{INDEX}

Mots-clés : sentiers, transports, trekking, portage, mobilité, tourisme, montagnes, dynamiques socio-spatiales

Keywords : Népal, tracks, transport, carrier, mobility, tourism, mountains, social change, Nepal

\section{AUTEUR}

\section{ISABELLE SACAREAU}

Isabelle SACAREAU est maître de conférences à l'Université de La Rochelle. Elle poursuit une réflexion théorique sur l'approche géographique du tourisme et des loisirs, tout en menant des études comparatives sur les grands massifs montagneux (Himalaya, Alpes, Haut-Atlas). Elle a publié en 1997 Porteurs de l'Himalaya aux éditions Belin, collection Mappemonde. 